\title{
Low treatment initiation among multi-drug resistant tuberculosis patients in Gauteng, South Africa, 2011
}

\author{
J.I. Ebonwu, ${ }^{1,2}$ K.S. Tint, ${ }^{1,2}$ C. Inekweazu ${ }^{3,4}$ \\ ${ }^{1}$ South African Field Epidemiology and Laboratory Training Programme, National Institute for \\ Communicable Diseases \\ ${ }^{2}$ School of Health Systems and Public Health, University of Pretoria, South Africa \\ ${ }^{3}$ Centre for Tuberculosis, National Institute for Communicable Diseases/ National Health Laboratory \\ Service, South Africa \\ ${ }^{4}$ Health Protection Agency, United Kingdom
}

\section{Corresponding author}

Joy I. Ebonwu

South African Field Epidemiology and Laboratory Training Programme

National Institute for Communicable Diseases

Private Bag X4, Sandringham, 2132, South Africa

Telephone: +27 118855317

Cellular: +27 824005588

Fax: +27118855339

E-mail: jodimegwu@yahoo.com

\section{SUMMARY}

Setting: Gauteng, South Africa

Objective: To determine treatment uptake among newly diagnosed multi-drug resistant tuberculosis (MDR-TB) patients and risk markers for non-initiation of treatment.

Design: A cross-sectional study was conducted including all newly diagnosed MDRTB patients in Gauteng province of South Africa in 2011. Socio-demographic and clinical characteristics of those that attended Sizwe hospital, the designated MDR-TB hospital, were extracted from their medical records. District health offices provided data on patients not seen at Sizwe hospital. Univariate and multivariate analysis were used to determine risk markers for non-initiation of treatment.

Results: Of the 942 newly diagnosed MDR-TB patients in Gauteng, only 593 (63\%) initiated treatment. Of these, $70(11.8 \%)$ did not maintain treatment up to the fourth month. Among the 349 (37\%) who did not initiate treatment, $31.2 \%$ died and $46.4 \%$ 
could not be accounted for. Referral for laboratory diagnosis from hospitals, health district of laboratory diagnosis, HIV infection and place of residence were independently associated with non-initiation of MDR-TB treatment.

Conclusion: Untreated patients continue the transmission of MDR-TB in communities. The study findings highlight the need to identify and target the causes of non-initiation of treatment in specific settings.

\section{Key words}

MDR-TB transmission, Patient tracing, untreated patients, Risk markers, death

\section{INTRODUCTION}

Successful treatment of multi-drug resistant tuberculosis (MDR-TB), defined as Mycobacterium tuberculosis resistance to isoniazid and rifampicin, depends on strict adherence to drug regimes of at least 24 months. Adherence to MDR-TB treatment is challenging for the patient because the duration of treatment is long, requires extensive hospitalization, and involves taking several medications, many of which produce uncomfortable adverse effects. ${ }^{1-2}$ Poor adherence, in turn, leads to a failure to cure MDR-TB, which increases the risk of poor patient outcomes. This leads to further spread of MDR-TB in communities with an associated increase in morbidity and mortality in the population. ${ }^{3-5}$

In addition to the health effects, treatment of MDR-TB is 50 to 200 times more expensive $^{6}$ to manage than drug susceptible TB. The challenge of managing MDRTB has been compounded by the emergence of extensively drug-resistant TB (XDRTB), defined as MDR-TB plus resistance to any fluoroquinolone and at least one of the injectable second-line drugs (amikacin, kanamycin, or capreomycin). Successful treatment of MDR-TB is therefore dependent on proper management of drug supplies, appropriate patient management and good patient adherence.

South Africa has the largest burden of MDR-TB in Africa and there has been a steady increase in the number of cases since $2004 .^{7-8}$ Gauteng is one of the nine 
provinces of South Africa and the smallest in terms of landmass. In 2011, it had a population of nearly 12.3 million, mainly living in its two major cities; Johannesburg and Pretoria, making it the most densely populated province in the country. In Gauteng, $1.4 \%$ of newly diagnosed TB and $5.5 \%$ of previously treated TB patients were diagnosed with MDR-TB as part of the last national drug resistance survey in $2001 .{ }^{9}$ There have been about 1000 new cases of MDR-TB diagnosed in the province every year between 2007 and $2011 .^{8}$ Ensuring that all patients diagnosed with MDR-TB are put on treatment is one of South Africa's long term strategies for addressing drug resistant TB (DR-TB). ${ }^{10}$

The non-initiation and delay in initiation of TB treatment have been described in a number of studies. ${ }^{11-13}$ In one study conducted in KwaZulu-Natal province, there was significant delay observed in initiating definitive management for MDR-TB ${ }^{14}$. Another study in the same province showed that only $67 \%$ of patients diagnosed between 1994 and 2004 had successful treatment outcomes. ${ }^{15}$ Results from these settings informed a review of the policy of centralised MDR-TB care in South Africa, in which all newly diagnosed MDR-TB patients were managed in a central hospital in each province. A new policy framework of decentralised and deinstitutionalised management of MDR-TB was adopted by the National Department of Health $(\mathrm{NDOH})$ in August 20117. However, because of the size and diversity of South Africa, the circumstances that lead to non-initiation may be different in different settings. Therefore a nationwide shift from one policy to another may in itself not address the underlying reasons leading to non-initiation of MDR-TB treatment. No study examining the reasons and risk markers for non-initiation of MDR-TB treatment has been found in South Africa.

The aim of this study was to determine treatment uptake among a full year's cohort of newly diagnosed MDR-TB patients in the Gauteng province of South Africa and to determine the proportion of the patients who maintained treatment up to the fourth month after treatment initiation. The study also examined the reasons for loss of MDR-TB patients along the treatment initiation pathway and risk markers for noninitiation of treatment. 


\section{STUDY POPULATION AND METHODS}

\section{Study setting}

Gauteng province is made up of six health districts; City of Johannesburg, Ekurhuleni Metro, City of Tshwane, West Rand, Sedibeng and Metsweding.

Once a diagnosis of MDR-TB is made in patients presenting to any clinic or hospital in Gauteng, they are referred to Sizwe Hospital for management. Sizwe is the hospital identified for the centralised management of all patients with MDR-TB in the province. Patients are admitted for an intensive phase of treatment lasting two months, and subsequently discharged to district clinics, to continue treatment after one culture negative conversion on a sputum sample. They are expected to report back to Sizwe Hospital after three months for review.

\section{Study design and population}

We conducted a cross-sectional study, with both descriptive and analytical components. All newly diagnosed MDR-TB patients, who attended government health facilities in Gauteng, with laboratory-confirmed results from $1^{\text {st }}$ January 2011 to $31^{\text {st }}$ December 2011, were included in the study.

\section{Sources of data}

A line-list of all laboratory-confirmed, newly diagnosed MDR-TB patients in Gauteng province in 2011 was obtained from the electronic repository of the South African National Health Laboratory Service. At Sizwe Hospital, the line list was used to match patients in the hospital's MDR-TB treatment register. The register is used to record and monitor the progress of all MDR-TB patients while on treatment, and also their treatment outcomes. The socio-demographic and clinical characteristics of these patients were extracted from the MDR-TB treatment register, their MDR-TB treatment cards and from the hospital admission database. The six district health offices in Gauteng were approached to provide data on patients not found in the hospital records at Sizwe. 


\section{Study variables}

The final study variables for which we had data were age, sex, race, employment status, place of residence, health districts, HIV status, referring health facilities, date of MDR-TB diagnosis, date of death, date of MDR-TB treatment initiation, MDR-TB treatment initiation status, reasons for not initiating treatment, maintenance of treatment up to the fourth month and reasons for not maintaining treatment..

\section{Data management and statistical analysis}

Deduplication of the line list obtained from the NHLS was carried out and the quality of data was assessed by comparing records in the MDR-TB treatment register and patient MDR-TB treatment card for $50 \%$ of the cases at Sizwe Hospital. The completed study inventory forms were manually checked for completeness and correctness. The study database was created using Epilnfo version 3.3.2 and analyzed using Stata $11.0\left(\right.$ StataCorp $\left.^{\circledR}\right)$.

Categorical data were summarized as proportions while median and inter-quartile range (IQR) were used to describe numeric data. Chi-squared test was used for comparison of categorical variables and Mann-Whitney-Wilcoxon ranked sums test was used to compare the median age of males and females. Univariate and multivariate analyses were used to determine risk markers for non-initiation of treatment. A p-value of less than 0.05 was regarded as statistically significant and $95 \%$ confidence interval $(\mathrm{Cl})$ was used to estimate precision.

\section{Ethics}

Ethics approval was obtained from the Faculty of Health Sciences Research Ethics Committee, University of Pretoria. Approval for the study was also granted by the Gauteng Department of Health, Sizwe Hospital management and the NHLS.

\section{RESULTS}

A total of 942 new MDR-TB patients were diagnosed by the NHLS laboratories in the Gauteng province of South Africa in 2011. The characteristics of these patients are described in table 1. 
Table1: Characteristics of the newly diagnosed MDR-TB patients - Gauteng, South Africa, 2011

Characteristics
Sex:
$\quad$ Male
Female

Age (years): Median (IQR)

\begin{tabular}{|c|c|}
\hline \multicolumn{3}{|c|}{$\mathbf{N}=942$} \\
\hline$n$ & $\%$ \\
\hline & \\
\hline & \\
\hline 43 & 50.2 \\
\hline
\end{tabular}

Age groups (years):
$<5$
$5-24$
$25-44$
$45-64$
$65+$

\section{HIV status:}

Positive

Negative

Previous TB treatment:
Yes
No

\section{Place of residence:}

Township

Prison

Informal settlement

Suburb

Town

Unknown

19
121
558
224
9

2.0

13.0

59.9

24.1

1.0

745

197

79.1

20.9

771
171

81.8

18.2

Referring facility:
Clinic
Correctional service
Hospital
Health district:
City of Johannesburg
City of Tshwane
Ekurhuleni
Metsweding
Sedibeng
West Rand

\begin{tabular}{cc}
\hline 695 & 73.8 \\
14 & 1.5 \\
52 & 5.5 \\
107 & 11.4 \\
61 & 6.5 \\
13 & 1.3
\end{tabular}

1.3

61.2

1.5

37.3

$352 \quad 37.3$

\begin{tabular}{c|c}
\hline 402 & 42.7 \\
195 & 20.7 \\
179 & 19.0 \\
12 & 1.3 \\
66 & 7.0 \\
88 & 9.3
\end{tabular}

The age of the MDR-TB patients ranged from 1 to 91 years and varied significantly by sex as males tended to be older than females $(p<0.01)$. The median age (in 
years) of males was 37.5 (95\% Cl: $36-39)$, compared to 33 (95\% Cl: $32-34)$ of the females. Most of the MDR-TB patients (59.9\%) were within the 25-44 years age group, as shown in Table 1.

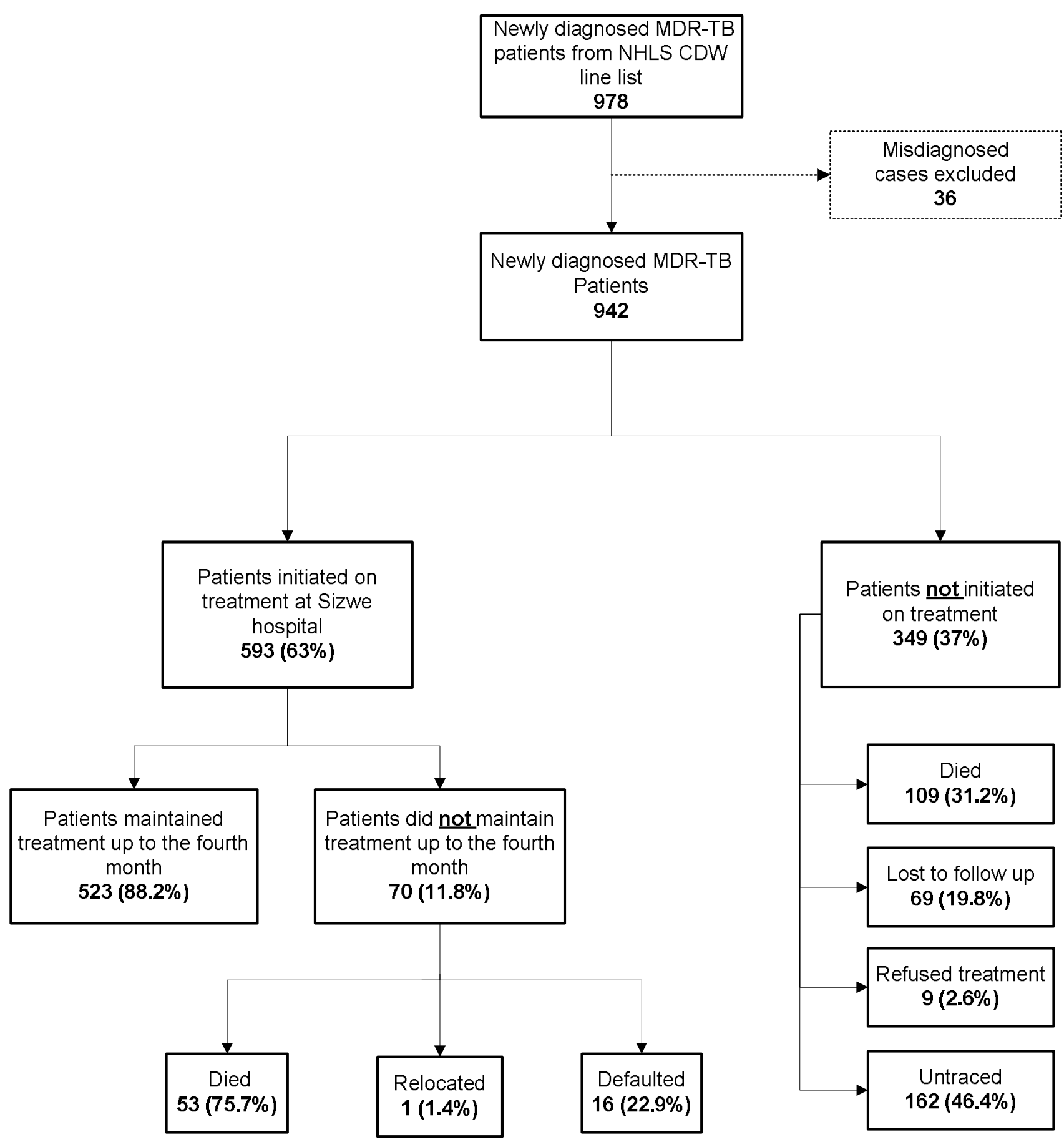

Figure 1: Study profile of the newly diagnosed MDR-TB patients - Gauteng, South Africa, 2011 
Of the 942 newly diagnosed MDR-TB patients in the province, only 593 (63\%) initiated treatment at Sizwe Hospital (Figure 1). The patients were initiated on treatment at a median of 10 days after the date of MDR-TB diagnosis. Most of the MDR-TB patients initiated on treatment were of black population group (94.7\%) and unemployed (66.9\%), HIV positive (82.1\%) and had previous history of TB treatment $(80.6 \%)$. Seventy $(11.8 \%)$ of the MDR-TB patients did not maintain treatment up to the fourth month as $75.7 \%(53 / 70)$ died at a median of 31 days after treatment initiation, $22.9 \%(16 / 70)$ defaulted and one 1.4\% (1/70) relocated. Co-infection with HIV was observed in $81.1 \%(43 / 53)$ of the patients who died and $81.3 \%(13 / 16)$ in those who defaulted. Majority of those who died $86.8 \%(46 / 53)$ and the defaulters $75 \%(12 / 16)$ had been previously treated for TB.

Table 2: Reasons for non-initiation of MDR-TB treatment and referring facilities

\begin{tabular}{|c|c|c|c|c|}
\hline \multirow[t]{2}{*}{ REASONS } & \multicolumn{3}{|c|}{ REFERRING FACILITIES } & \multirow{2}{*}{$\begin{array}{c}\text { TOTAL } \\
\text { (\%) }\end{array}$} \\
\hline & Clinics (\%) & $\begin{array}{c}\text { Correctional services } \\
\qquad(\%)\end{array}$ & Hospitals (\%) & \\
\hline Death & $21(19.3)$ & $1(0.9)$ & $87(79.8)$ & $109(31.2)$ \\
\hline Lost to follow up & $38(55.1)$ & $1(1.4)$ & $30(43.5)$ & $69(19.8)$ \\
\hline Treatment refusal & $7(77.8)$ & 0 & $2(22.2)$ & $9(2.6)$ \\
\hline Untraced & $84(51.9)$ & 0 & 78 (48.1) & $162(46.4)$ \\
\hline TOTAL (\%) & $150(43)$ & $2(0.6)$ & $197(56.4)$ & 349 \\
\hline
\end{tabular}

Of the 349/942 (37\%) that did not initiate MDR-TB treatment (Figure 1), $293(84 \%)$ were previously treated for TB and 258 (73.9\%) were HIV positive. Over half (56.5\%) of the patients were referred for laboratory diagnosis from hospitals. Of the 349, 109 $(31.2 \%)$ died prior to initiation of treatment while $162(46.4 \%)$ could not be traced by the health system (Figure 1). The patients died at a median interval, from MDR-TB diagnosis, of 17.5 days. Of those who died prior to treatment initiation, $79.8 \%$ (87/109) were referred for laboratory diagnosis from hospitals (Table 2) and $74.3 \%$ (81/109) were co-infected with HIV (Table 3). 
Table 3: Reasons for non-initiation of MDR-TB treatment and HIV status

\begin{tabular}{|l|c|c|c|}
\hline REASONS & \multicolumn{2}{|c|}{ HIV STATUS } & TOTAL \\
\hline Death & Positive (\%) & Negative (\%) & (\%) \\
\hline Lost to follow up & $81(74.3)$ & $28(25.7)$ & $109(31.2)$ \\
\hline Treatment refusal & $56(81.2)$ & $13(18.8)$ & $69(19.8)$ \\
\hline Untraced & $8(88.9)$ & $1(11.1)$ & $9(2.6)$ \\
\hline TOTAL (\%) & $113(69.8)$ & $49(30.2)$ & $162(46.4)$ \\
\hline
\end{tabular}

Table 4 shows the risk markers associated with non-initiation of MDR-TB treatment. Patients referred from a hospital were eight times $(95 \% \mathrm{Cl} 1.8-37.8)$ more likely not to initiate treatment than patients referred from a clinic. Of the six health districts in Gauteng, patients residing in the City of Tshwane $(95 \% \mathrm{Cl} 1.6-3.5)$ and Ekurhuleni $(95 \%$ Cl 1.4 - 3.3) were two times more likely not to initiate treatment respectively, compared to City of Johannesburg. Using the townships, where most patients lived, as reference for place of residence, our study showed that those who reside in the central business districts in Gauteng were two times (95\% Cl 1.1 - 3.6) more likely not to initiate treatment while those residing in the prisons $(0.1(95 \% \mathrm{Cl} 0.2-0.5))$, informal settlements $(0.4(95 \% \mathrm{Cl} 0.2-0.8))$ and in suburbs $(0.3(95 \% \mathrm{Cl} 0.2-0.6))$ were less likely not to initiate treatment respectively, as well as those with HIV infection $(0.6(95 \% \mathrm{Cl} 0.4-0.8))$.

\section{DISCUSSION}

Of the 942 newly diagnosed MDR-TB patients in Gauteng province in 2011, only $63 \%$ were initiated on treatment at Sizwe Hospital. The $37 \%$ who did not initiate MDR-TB treatment in our study population indicates a large gap between the number of diagnosed MDR-TB cases and number started on treatment. This gap was observed across all the health districts in our study. These findings in Gauteng are similar to the national picture. ${ }^{16}$ The implication of delayed initiation or noninitiation of MDR-TB treatment is the risk of increased morbidity and mortality for the 
Table 4: Risk markers for non-initiation of MDR-TB treatment in the study population

\begin{tabular}{|c|c|c|c|c|c|c|}
\hline \multirow[t]{2}{*}{ Variable } & \multicolumn{2}{|c|}{$\begin{array}{l}\text { Initiated MDR-TB treatment } \\
\text { n (\%) }\end{array}$} & \multicolumn{2}{|c|}{ Univariate analysis } & \multicolumn{2}{|c|}{ Multivariate analysis } \\
\hline & $\begin{array}{c}\text { No } \\
349(37)\end{array}$ & $\begin{array}{c}\text { Yes } \\
593(63)\end{array}$ & OR & $95 \% \mathrm{Cl}$ & AOR & $95 \% \mathrm{Cl}$ \\
\hline $\begin{array}{l}\text { Sex } \\
\text { Male } \\
\text { Female }\end{array}$ & $\begin{array}{l}166(47.6) \\
183(52.4)\end{array}$ & $\begin{array}{l}307(51.8) \\
286(48.2)\end{array}$ & $\begin{array}{l}0.8 \\
\text { Ref }\end{array}$ & $0.6-1.1$ & & \\
\hline $\begin{array}{l}\text { Age groups (years) } \\
<5 \\
5-24 \\
25-44 \\
45-64 \\
65+\end{array}$ & $\begin{array}{c}6(1.8) \\
37(10.9) \\
223(66.0) \\
67(19.8) \\
5(1.5)\end{array}$ & $\begin{array}{c}13(2.2) \\
84(14.1) \\
335(56.5) \\
157(26.5) \\
4(0.7)\end{array}$ & $\begin{array}{l}\text { Ref } \\
0.9 \\
1.4 \\
0.9 \\
2.7\end{array}$ & $\begin{array}{c}0.3-2.7 \\
0.5-3.9 \\
0.3-2.5 \\
0.5-13.9\end{array}$ & & \\
\hline $\begin{array}{l}\text { Previous } \\
\text { TB treatment } \\
\text { Yes } \\
\text { No }\end{array}$ & $\begin{array}{c}293(84.0) \\
56(16.0)\end{array}$ & $\begin{array}{l}478(80.6) \\
115(19.4)\end{array}$ & $\begin{array}{l}1.3 \\
\text { Ref }\end{array}$ & $0.9-1.8$ & & \\
\hline $\begin{array}{l}\text { Place of residence } \\
\text { Township } \\
\text { Prison } \\
\text { Informal settlement } \\
\text { Suburb } \\
\text { Town } \\
\text { Unknown }\end{array}$ & $\begin{array}{c}266(76.2) \\
2(0.6) \\
10(2.9) \\
22(6.3) \\
36(10.3) \\
13(3.7)\end{array}$ & $\begin{array}{c}429(72.4) \\
12(2.0) \\
42(7.1) \\
85(14.3) \\
25(4.2) \\
0\end{array}$ & $\begin{array}{c}\text { Ref } \\
0.3 \\
0.4 \\
0.4 \\
2.3 \\
\text { omitted }\end{array}$ & $\begin{array}{l}0.6-1.2 \\
0.2-0.8 \\
0.3-0.7 \\
1.4-3.9\end{array}$ & $\begin{array}{l}\text { Ref } \\
0.1 \\
0.4 \\
0.3 \\
1.9\end{array}$ & $\begin{array}{l}0.2-0.5 \\
0.2-0.8 \\
0.2-0.6 \\
1.1-3.6\end{array}$ \\
\hline $\begin{array}{l}\text { Health district } \\
\text { City of Johannesburg } \\
\text { City of Tshwane } \\
\text { Ekurhuleni } \\
\text { Metsweding } \\
\text { Sedibeng } \\
\text { West Rand }\end{array}$ & $\begin{array}{c}140(40.1) \\
87(24.9) \\
76(21.8) \\
3(0.9) \\
16(4.6) \\
27(7.7)\end{array}$ & $\begin{array}{c}262(44.2) \\
108(18.2) \\
103(17.4) \\
9(1.5) \\
50(8.4) \\
61(10.3\end{array}$ & $\begin{array}{l}\text { Ref } \\
1.5 \\
1.4 \\
0.6 \\
0.6 \\
0.8\end{array}$ & $\begin{array}{l}1.1-2.1 \\
0.9-1.9 \\
0.2-2.3 \\
0.3-1.1 \\
0.5-1.4\end{array}$ & $\begin{array}{l}\text { Ref } \\
2.3 \\
2.1 \\
1.4 \\
0.9 \\
1.2\end{array}$ & $\begin{array}{l}1.6-3.5 \\
1.4-3.3 \\
0.4-5.5 \\
0.5-1.7 \\
0.7-2.1\end{array}$ \\
\hline
\end{tabular}

affected individuals and a persisting risk of further transmission of MDR-TB in the community. ${ }^{11,13,17}$ 
We observed that $17.2 \%$ (162/942) of our cohort of MDR-TB patients were known to have died. Of these, $67.3 \%(109 / 162)$ did not initiate treatment while $32.7 \%(53 / 162)$ initiated treatment. In addition to those known to have died, close to half $(46.4 \%)$ of the untreated MDR-TB patients could not be accounted for by the health system and it can be assumed that a proportion also died. Good implementation of the intensified case finding strategy for MDR-TB as per NDOH guidelines ${ }^{10}$ could reduce deaths.

In our study, referral for laboratory diagnosis from hospitals was a significant risk marker for non-initiation of MDR-TB treatment suggesting that hospitals may be less effective than clinics in following up newly diagnosed MDR-TB patients. Of those who died prior to treatment initiation, $79.8 \%$ were referred for laboratory diagnosis from hospitals. This suggests that delays in following up patients newly diagnosed with MDR-TB in hospitals ${ }^{13,18}$ and underlying co-morbidities ${ }^{19-20}$ may be associated with an increased risk of death before MDR-TB treatment initiation. Prioritising patients referred from hospitals for follow-up and ensuring that they enter the care pathway would likely prevent deaths occurring before treatment initiation.

Additionally, the diagnosis of MDR-TB in two of Gauteng's districts, City of Tshwane and Ekurhuleni, were also significantly associated with non-initiation of treatment. These districts are the most urbanised and industrialised in the province, with academic and tertiary hospitals. Further investigation is required into why these patients did not initiate treatment.

Adjusting for other variables, co-infection with HIV was associated with the initiation of MDR-TB treatment. It is not clear why this is the case but it is possible that the integration of TB/HIV services could have made the referral of the HIV positive patients for MDR-TB treatment easier, as the two health programmes are involved with the care of the same patient. However, co-infection with HIV was high among the MDR-TB patients who died prior to treatment initiation (74.3\%) and those who died within four months of treatment $(81.1 \%)$.

We also found that patients resident in prisons, informal settlements and in the suburbs were more likely to initiate treatment than those resident in the townships. 
Patients in the prisons are more likely to take up treatment due to its confined nature and strict rules. The incentives (such as social grants) provided to those on MDR-TB treatment could be the reason for those in informal settlements initiating treatment and relative affluence of those residing in the suburbs could explain their access to care.

Poor access to treatment and delayed treatment initiation were some of the barriers to successful MDR-TB treatment in South Africa. Decentralised MDR-TB treatment in the South African provinces of Western Cape ${ }^{21}$ and KwaZulu-Natal ${ }^{22-23}$, demonstrated increased case detection, decreased time to diagnosis and treatment initiation, as well as improved treatment outcomes, leading to the adoption of a decentralised model of MDR-TB management in 2011.

There is limited evidence, however on the benefits of decentralisation in Gauteng, given its unique characteristics in density and the preponderance of tertiary care settings. There is also little evidence that the underlying causes of non-initiation of MDR-TB treatment had been investigated prior to this study and any attempts made to address them. As the decentralization of MDR-TB is already being implemented, this study therefore provides baseline information with which to evaluate the impact of the introduction of decentralisation of the management of patients with MDR-TB in Gauteng.

Our study had some limitations. It relied on the review of records and did not account for the interval between specimen collection and diagnosis. Positive TB smear or/and culture results, between 2007 and 2011, were used as proxy for previous TB treatment. Those who could have had TB prior to 2007 were possibly excluded. In retrospect, we should have considered the distance from patient residence to Sizwe hospital as a variable for reasons for non-initiation of treatment, although distance may not be as much an issue in Gauteng as in other provinces in South Africa due to close proximity. 


\section{CONCLUSION}

In this study, 63\% treatment uptake among newly diagnosed MDR-TB patients is far from ideal because untreated patients continue the transmission of MDR-TB in communities. The findings of the study highlight the need to identify and target the causes of non-initiation of MDR-TB treatment, such as the improvement of patient tracing and referral. Determining the risk markers for non-initiation of MDR-TB treatment would aid health providers in improving diagnosis by addressing these challenges and treatment efforts. In Gauteng, we recommend the intensification of follow-up of MDR-TB cases, particularly those identified in hospitals.

\section{ACKNOWLEDGEMENTS}

The authors contributed substantially to the conception and design, acquisition and analysis of data, as well as critical review of the final report. They wish to acknowledge $\mathrm{Dr}$ Gerrit Coetzee (former director of the National TB reference laboratory, South Africa) for his role in the development of the study protocol and the following institutions for their support towards the study: South African Field Epidemiology and Laboratory Training Programme; Centre for Tuberculosis, NICDNHLS; School of Health Systems and Public Health, University of Pretoria; Gauteng Department of Health and the district health offices. There is no conflict of interest, as no funding was received to conduct the study. 


\section{REFERENCES}

1. Weyer K, Van der Walt M, Kantor P. Managing multi-drug resistant tuberculosis: Legal implications. MRC Policy brief 2006. http:// www.mrc.ac.za/policybriefs/managing TB.pdf. Accessed February 2013.

2. Shin SS, Pasechnikov AD, Gelmanova IY et al. Adverse reactions among patients being treated for MDR-TB in Tomsk, Russia. Int J Tuberc Lung Dis. 2007;11(12):1314-1320.

3. Cox HS, Kalon S, Allamuratova $S$ et al. Multidrug-resistant tuberculosis treatment outcomes in Karakalpakstan, Uzbekistan: Treatment complexity and XDR-TB among treatment failures. PLoS One 2007; 2(11):e1126.

4. Shean KP, Willcox PA, Siwendu SN et al. Treatment outcome and follow-up of multidrug-resistant tuberculosis patients, West Coast/Winelands, South Africa, 1992-2002. Int J Tuberc Lung Dis. 2008; 12(10):1182-1189.

5. Kliiman K, Altraja A. Predictors of poor treatment outcome in multi- and extensively drug-resistant pulmonary TB. Eur Respir J. 2009; 33(5):10851094.

6. World Health Organization. Multidrug and extensively drug-resistant TB (M/XDR-TB): 2010 global report on surveillance and response. Geneva, WHO report. WHO/HTM/TB/2010.3.

7. National Department of Health. Multi-drug resistant tuberculosis: A policy framework on decentralized and deinstituitionalized management for South Africa. Pretoria: South Africa, 2011. 
8. Koornhof H, Ihekweazu C, Erasmus L, Coetzee G. Update on Corporate Data Warehouse-derived MDR- and XDR-TB statistics for eight provinces in South Africa, January 2007 to $30^{\text {th }}$ June 2011. Communicable Dis Surveillance Bull. 2011; 9(1):68-72.

9. Weyer K, Lancaster J, Brand J, Van der Walt M, Levin J. Survey of tuberculosis drug resistance in South Africa: 2001-2002: Medical Research Council of South Africa 2004.

10. National Department of Health. Management of drug-resistant tuberculosis: policy guidelines. Pretoria: South Africa, 2011.

11. Yimer S, Bjune G, Alene G. Diagnostic and treatment delay among pulmonary tuberculosis patients in Ethiopia: a cross sectional study. BMC Infect Dis. 2005; 5:112.

12. Chern JP, Chen DR, Wen TH. Delayed treatment of diagnosed pulmonary tuberculosis in Taiwan. BMC Public Health. 2008; 8:236.

13. Farah MG, Rygh JH, Steen TW, Selmer R, Heldal E, Bjune G. Patient and health care system delays in the start of tuberculosis treatment in Norway. BMC Infect Dis. 2006; 6:33.

14. Narasimooloo R, Ross A. Delay in commencing treatment for MDR TB at a specialised TB treatment centre in KwaZulu-Natal. S Afr Med J. 2012; 102(6):360-362.

15. Loveday $\mathrm{M}$, Wallengren $\mathrm{K}$, Voce $\mathrm{A}$ et al. Comparing early treatment outcomes of MDR-TB in a decentralised setting with a centralised setting in KwaZuluNatal, South Africa. Int J Tuberc Lung Dis. 2012; 16(2): 209-215. 
16. World Health Organization. South African Tuberculosis profile 2011. http:// www.who.int/tb/data. Accessed September 2011.

17. Golub JE, Bur S, Cronin WA et al. Delayed tuberculosis diagnosis and tuberculosis transmission. Int J Tuberc Lung Dis. 2006; 10(1):24-30.

18. Meintjes G, Schoeman H, Morroni C, Wilson D, Maartens G. Patient and provider delay in tuberculosis suspects from communities with a high HIV prevalence in South Africa: a cross-sectional study. BMC Infect Dis. 2008; 8:72.

19. Lee $\mathrm{CH}$, Lee $\mathrm{MC}$, Lin $\mathrm{HH}$ et al. Pulmonary tuberculosis and delay in antituberculous treatment are important risk factors for chronic obstructive pulmonary disease. PLoS One. 2012; 7(5):e37978.

20. Cain KP, Anekthananon T, Burapat $C$ et al. Causes of death in HIV-infected persons who have tuberculosis, Thailand. Emerg Infect Dis. 2009; 15(2):25864.

21. Médecins Sans Frontiéres. Scaling up diagnosis and treatment of drugresistant tuberculosis in Khayelitsha, South Africa: An integrated, communitybased approach, March 2011. http//: www.msf.org.za. Accessed October 2011.

22. Brust JC, Gandhi NR, Carrara H, Osburn G, Padayatchi N. High treatment failure and default rates for patients with multidrug-resistant tuberculosis in KwaZulu-Natal, South Africa, 2000-2003. Int J Tuberc Lung Dis. 2010; 14(4):413-419.

23. Shenoi S, Heysell S, Moll A, Friedland G. Multidrug-resistant and extensively drug-resistant tuberculosis: consequences for the global HIV community. Curr Opin Infect Dis. 2009; 22(1):11-17. 